\title{
Edge-distinguishing of star-free graphs
}

\author{
Aleksandra Gorzkowska* Ernest Kargul \\ Szymon Musiał Katarzyna Pal \\ Department of Discrete Mathematics \\ AGH University of Science and Technology \\ Kraków, Poland \\ agorzkow@agh.edu.pl \\ Submitted: Feb 18, 2020; Accepted: Jul 23, 2020; Published: Aug 7, 2020 \\ (C) The authors. Released under the CC BY-ND license (International 4.0).

\begin{abstract}
The distinguishing index $D^{\prime}(G)$ of a graph $G$ is the least number $k$ such that $G$ has an edge colouring with $k$ colours that is only preserved by the trivial automorphism. Pilśniak proved that a connected, claw-free graph has the distingushing index at most three. In this paper, we show that the distingushing index of a connected, claw-free graph with at least six vertices is bounded from above by two. We also consider more general graphs in this sense. Namely, we prove that if $G$ is a connected, $K_{1, s}$-free graph of order at least six, then $D^{\prime}(G) \leqslant s-1$.
\end{abstract}

Mathematics Subject Classifications: 05C25, 05C80, 03E10

\section{Introduction}

The notation follows the standard terminology as in [2]. We consider edge colourings, not necessarily proper, of simple, connected graphs. We say that an edge colouring $c$ breaks an automorphism $\varphi$ of the graph $G$ if there exists an edge such that its colour is different from the colour of its image under the automorphism's action. Otherwise, we say that the automorphism $\varphi$ preserves the colouring $c$. The distinguishing index of a graph $G$, denoted by $D^{\prime}(G)$, is the least number $k$ such that $G$ admits an edge colouring with $k$ colours breaking every nontrivial automorphism of the graph $G$.

The definition of the distinguishing index, introduced by Kalinowski and Pilśniak in [5], was inspired by the distinguishing number which was defined for general vertex colourings by Albertson and Collins [1]. The distinguishing number of a graph $G$, denoted by $D(G)$, is the least number of colours used in a vertex colouring breaking every nontrivial

*The research was partially supported by the Polish Ministry of Science and Higher Education. 
automorphism of the graph $G$. Clearly, $D(G)=D^{\prime}(G)=1$ for all graphs with no nontrivial automorphism and the distinguishing index is not defined for graphs with $K_{2}$ as a connected component. Moreover, the distinguishing number as well as the distinguishing index of cycles $C_{3}, C_{4}$ and $C_{5}$ equals three, while for cycles of order $n \geqslant 6$ we have $D\left(C_{n}\right)=D^{\prime}\left(C_{n}\right)=2$.

Kalinowski and Pilśniak in their paper proved a general upper bound on the distinguishing index of connected graphs. Namely, they proved that if $G$ is a connected graph with at least three vertices and maximal degree $\Delta(G)$, then $D^{\prime}(G) \leqslant \Delta(G)$, with the exception of three cycles $C_{3}, C_{4}$ and $C_{5}$. Moreover, they proved that this bound is best possible in a general case. They showed that there exist classes of trees, called symmetric and bisymmetric trees, for which the equality in the upper bound is achieved. Therefore, the research in this field is focused on finding better upper bounds on the distinguishing index for a given class of graphs.

The main motivation for our research was a result of Pilśniak for the class of claw-free graphs. We consider graphs which do not contain a star $K_{1, s}$ as an induced subgraph. We call such graphs $K_{1, s^{-}}$free graphs. The star $K_{1,3}$ is usually called a claw, and the graph without a claw as an induced subgraph is called a claw-free graph. Pilśniak proved the following.

Theorem 1. [6] Let $G$ be a connected, claw-free graph. Then

$$
D^{\prime}(G) \leqslant 3
$$

In the same paper she also conjectured that for graphs of sufficiently large order, this bound could be improved.

Conjecture 2. [6] There exists a constant $n_{0} \in \mathbb{N}_{+}$such that every connected, claw-free graph $G$ of order $n \geqslant n_{0}$ satisfies

$$
D^{\prime}(G) \leqslant 2 \text {. }
$$

Claw-free graphs have been extensively studied over the years, many of the obtained results were compiled in a survey by Faudree, Flandrin and Ryjáček in [3]. The goal of our research was to obtain a sharp bound for the distinguishing index of claw-free graphs, thus proving the conjecture posed by Pilśniak. We also consider more general case of $K_{1, s}$-free graphs. The main theorem of this paper is the following.

Theorem 3. Let $G$ be a connected, $K_{1, s}$-free graph with $s \geqslant 3$ and $|G| \geqslant 6$. Then

$$
D^{\prime}(G) \leqslant s-1
$$

The case for claw-free graphs is proved in Section 3 while the case for $s \geqslant 4$ is proved in Section 4. 


\section{Preliminaries}

\subsection{Neighbourhood lemmas and definitions}

By $N(x)$ we denote the neighbourhood of a vertex $x$, and by $N[x]$ we denote its closed neighbourhood. We say that a vertex $x \in V(G)$ is fixed by a colouring $c$ if for each automorphism $\varphi \in \operatorname{Aut}(G)$ preserving the colouring $c$, we have $\varphi(x)=x$. Whenever it does not cause ambiguity, we say that $x$ is fixed. Given a subset of vertices $S \subseteq V(G)$, we say that $S$ is fixed setwise by a colouring $c$ if for each automorphims $\varphi \in \operatorname{Aut}(G)$ preserving the colouring $c$, we have for each vertex $x \in S$ that $\varphi(x) \in S$. Observe that if a vertex $x$ is fixed, then the neighbourhood of $x$ is fixed setwise. Clearly, if every vertex in the graph $G$ is fixed by a colouring $c$, then $c$ breaks every nontrivial automorphism of $G$.

We write $a P b$ to indicate a path $P$ joining vertices $a$ and $b$ in a graph $G$. Analogously, for a cycle $C$ in $G$ such that $a, b \in V(C)$ we write $a C b$ to indicate one of the two paths consisting of the edges of $C$ joining the vertices $a$ and $b$. We denote the distance on the cycle $C$ of the vertices $a$ and $b$, that is the number of edges of the shortest path joining $a$ and $b$ that consists only of the edges of the cycle $C$, by $\operatorname{dist}_{C}(a, b)$.

An important result for our studies is a theorem proved by Fouquet in [4]. Recall that $\alpha(G)$ denotes the independence number of the graph $G$, i.e., the number of vertices in the largest independent set.

Theorem 4. [4] Let $G$ be a connected claw-free graph with $\alpha(G) \geqslant 3$. Then every vertex $v$ of $G$ satisfies one (and only one) of the following conditions:

1. $N(v)$ is covered by two complete graphs,

2. $N(v)$ contains an induced $C_{5}$.

The first step in order to prove Theorem 3 can be seen as a generalization of this result onto $K_{1, s}$-free graphs.

Let $G$ be a graph. A path cover of $G$ is a set of paths $\mathcal{P}=\left\{P_{i}: i \in I\right\}$ such that every vertex of $G$ belongs to exactly one path from $\mathcal{P}$. Clearly every graph $G$ has a path cover as it is enough to take $|G|$ single-vertex paths. For each of those paths we select one of its end-vertices and we call it a first vertex of this path. A minimal path cover of $G$ is a path cover of $G$ with the least number of paths.

Lemma 5. Let $G$ be a connected $K_{1, s}$-free graph with $s \geqslant 3$, and let $x y$ be an edge of $G$. If $A \subseteq N(x)$ and $B \subseteq N(x) \backslash N[y]$, then the following two conditions hold:

1. There exists a path cover of $G[A]$ with at most $s-1$ paths.

2. There exists a path cover of $G[B]$ with at most $s-2$ paths.

Proof. 1. Assume that a minimal path cover of $G[A]$ consists of at least $s$ paths. Notice that any two first vertices of these paths cannot be connected by an edge. Otherwise 
we could construct a path cover with smaller number of paths, which is a contradiction. However, if no two of these vertices are connected, and all are connected with $x$, then those vertices together with $x$ induce a star $K_{1, s}$ in $G$. Which is a contradiction.

2. Once again assume to the contrary that a minimal path cover of $G[B]$ consists of at least $s-1$ paths. By identical reasoning as before, there are no edges connecting the first vertices of these paths. Now, these vertices together with vertices $x$ and $y$ induce in $G$ a star with at least $s+1$ vertices, which is a contradiction.

\subsection{Algorithm}

In this section we introduce an algorithm that extends a given partial colouring of the edges of a graph $G$ into a complete edge colouring of $G$. The algorithm has properties that will be useful for us in the proof of Theorem 3 and will simplify this proof. We present those important properties in the form of some observations.

\section{ALGORITHM.}

IN: $G$ - a connected $K_{1, s}$ free graph

$H \subseteq V(G): G[H]$ is connected and $|H| \geqslant 2, \exists v \in H: N(v) \subseteq H$,

$c: E(G[H]) \rightarrow\{1, \ldots, s-1\}$,

OUT: $c^{\prime}: E(G) \rightarrow\{1, \ldots, s-1\}$

1. Label the vertices of $G$ with natural numbers, beginning with the vertex $v \in H$ such that $N(v) \subseteq H$ and continuing in such a way, that the graph induced by vertices with labels $\{1,2, \ldots, i\}$ is connected for every $i \in\{1,2, \ldots,|V(G)|\}$. Let $S=H$, $H^{\prime}=H,\left.c^{\prime}\right|_{G[H]}=c$ and $c^{\prime}(e)=1$ for each $e \notin E(G[H])$.

2. Take a vertex from $H^{\prime}$ with the smallest label and call it $a$.

3. If $N[a] \backslash S=\emptyset$, then substitute $H^{\prime}=H^{\prime} \backslash\{a\}$ and go to step 2 .

4. Take a minimal path cover of $G[N[a] \backslash S]$. By Lemma 5, this path cover consists of at most $s-2$ paths. Order these paths with natural numbers starting with 1 . Colour every edge of the $i$-th path, as well as an edge between the first vertex of that path and the vertex $a$, with colour $i+1$.

5. Substitute $H^{\prime}=H^{\prime} \cup(N(a) \backslash S)$ and $S=S \cup N(a)$.

6. If $S \neq V(G)$, go to step 2 .

7. Return $c^{\prime}$.

The colouring $c^{\prime}$ returned by the ALGORITHM is such that every vertex outside of the input set of vertices $H$ has exactly one path to $H$ which does not contain an edge coloured with 1. Throughout the ALGORITHM, $S$ is the set of all the vertices that the resulting colouring has reached so far and $H^{\prime}$ is the set of the vertices whose neighbourhood we still 
have to potentially consider. The labeling of the vertices of $G$ ensures that each time the ALGORITHM takes a vertex $a \in H^{\prime}$ that has neighbours outside of $S$, the vertex $a$ also has at least one neighbour $b$ such that $N(b) \subseteq S$. Therefore, by part 2 of Lemma 5 there exists a path cover of $G[N(a) \backslash S]$ consisting of at most $s-2$ paths. The ALGORITHM then recolours the edges $G[N[a] \backslash S]$ so that no cycle without edges coloured with colour 1 is ever created. The vertices of $N(a)$ have been reached and so we add them to the set $S$ as well as the set $H^{\prime}$. The ALGORITHM chooses another vertex from the modifed set $H^{\prime}$ and finishes after all vertices of $G$ are added to $S$.

The following observations about the colouring output by the ALGORITHM are particularly important for us in further considerations.

Observation 6. For each $v \in V(G) \backslash H$, there exists $u \in V(G)$ such that $c^{\prime}(v u) \neq 1$.

Proof. All we need to observe is that every vertex $v \in V(G) \backslash H$ is contained in a path from some minimal path cover at some point of the ALGORITHM. The edges of such a path are coloured with a colour different from 1.

Observation 7. Let $C$ be a cycle in $G$. If $C$ contains a vertex from the set $V(G) \backslash H$, then at least one of the edges of $C$ is coloured with 1.

Proof. Obviously, after step 1 of the ALGORITHM is executed, every vertex of the set $V(G) \backslash H$ is incident only to edges coloured with colour 1 . Therefore, it suffices to prove that every time the ALGORITHM recolours the edges of the graph $G$, there will not appear a cycle with no edge coloured with 1.

Assume that in some step of the ALGORITHM a cycle $C$ with no edges coloured with 1 is created. Then some of the edges of $C$ must have been recoloured in this step. Thus the end-vertices of the recoloured edges belong to the closed neighbourhood of the vertex $a \in H^{\prime} \subseteq S$ considered in this step.

Consider the longest path $P$ of edges of the cycle $C$ that were recoloured in the last step. Let $k$ be the length of this path and denote by $x_{i}$ for $i \in\{0,1, \ldots, k\}$ the vertices of $P$, such that $x_{i} x_{i+1}, i \in\{0,1, \ldots, k-1\}$, is an edge of $C$ that was recoloured. Since the other edge of $C$ with end-vertex $x_{0}$ was not recoloured and is coloured with a colour different than 1 , then $x_{0} \in H^{\prime}$. Analogously, $x_{k} \in H^{\prime}$.

Notice that for all $i \in\{1, \ldots, k-1\}$ the vertices $x_{i}$ and $x_{i+1}$ belong to the same path from the minimal path cover. Otherwise, the edge $x_{i} x_{i+1}$ would be coloured with 1 . Since the edge $x_{0} x_{1}$ was recoloured, then $x_{0}=a$ and $x_{0}$ is the first vertex of some path of the minimal path cover. Therefore, either the edge $x_{k-1} x_{k}$ is coloured with 1 or $x_{k-1}=x_{0}$. In both cases we get a contradiction which ends the proof.

Now we can prove the following theorem.

Theorem 8. Assume that $H \subseteq V(G)$ such that $|H| \geqslant 2, G[H]$ is connected, there exists a vertex $v \in H$ such that $: N(v) \subseteq H$ and $c: E(G[H]) \rightarrow\{1,2, \ldots, k\}$. Let $c^{\prime}$ be the colouring returned by the ALGORITHM. If $v$ is fixed by $c^{\prime}$ for each $v \in H$, then every vertex in the graph $G$ is also fixed. 
Proof. We show that each time the ALGORITHM modifies set $S$, every vertex of the new set $S$ is fixed by the colouring $c^{\prime}$. We use induction on the number of times the ALGORITHM executes step 4.

Let $a$ be the first vertex such that $N(a) \backslash S \neq \emptyset$ and let $\mathcal{P}=\left\{P_{i}: i \in I\right\}$ be the minimal path cover of $G[N(a) \backslash S]$. Moreover, let $x_{i}$ be the first vertex of the path $P_{i}$. Since $a \in H$, then it is fixed by the assumption. Furthermore, $x_{i}$ is the only vertex at distance one from the set $H$ connected to $a$ by an edge coloured with $i+1$. Therefore $x_{i}$ is fixed for all $i \in I$. Take the successor of $x_{i}$ on the path $P_{i}$. It is also fixed, as it is the only vertex of $N(a) \backslash H$ joined to $x_{i}$ by an edge coloured with $i+1$. By the same argumentation the remaining vertices of each path $P_{i}$ are fixed. Therefore, every vertex of the set $H \cup N(a)$ is fixed by $c^{\prime}$.

Consider now the $k$ th time the ALGORITHM executes step 4. By induction hypothesis, all the vertices of $S$ at this point are fixed. In particular, currently considered vertex $a$ is fixed. The identical reasoning as above shows that all vertices in the set $N(a)$ are fixed.

\section{Claw-free graphs}

In this section we prove Theorem 3 for the case where $G$ is a claw-free graph. Thus, proving the conjecture posed by Pilśniak in [6]. We cite one more result obtained by Pilśniak, which gives the bound on the distinguishing index of the class of traceable graphs.

Theorem 9. [6] Let $G$ be a traceable graph of order $n \geqslant 7$. Then

$$
D^{\prime}(G) \leqslant 2 \text {. }
$$

This result is very useful for us, as it allows us to shorten our proof in one of the cases.

Theorem 10. Let $G$ be a connected, claw-free graph of order at least six. Then

$$
D^{\prime}(G) \leqslant 2
$$

Proof. We divide the proof into two parts. We start by considering the case where $G$ is a 2-connected graph. Let $C$ be the longest cycle in $G$.

Assume that $C$ is Hamiltonian. If the graph $G$ has at least seven vertices, then the claim follows from Theorem 9. Otherwise, the graph has exactly six vertices. We examine all possible maximal degrees of such a graph. If $\Delta(G)=2$, then $G=C_{6}$ and it is known that $D^{\prime}\left(C_{6}\right)=2$. Otherwise, since $\Delta(G) \in\{3 ; 4 ; 5\}$, then $G$ has the only asymmetric graph on six vertices as a spanning subgraph. We colour the edges of this spanning subgraph with colour 1 and the remaining edges with colour 2. Such a colouring breaks every nontrivial automorphism of $G$.

Now, if $C$ is not a Hamiltonian cycle, then there exists a vertex $v \in V(C)$ that has a neighbour, call it $w$, outside of $C$. We consider one of the two orientations of the cycle $C$ and call the vertices before and after $v$ on the cycle $v^{-}$and $v^{+}$, respectively. From maximality of $C, w$ cannot be adjacent to $v^{-}$nor $v^{+}$. Since $G$ is a claw-free graph, then 
$v^{-} v^{+}$is an edge of $G$. We denote by $C^{\prime}$ the cycle obtained from $C$ by removing $v$ and adding the edge $v^{-} v^{+}$.

$$
V\left(C^{\prime}\right)=V(C) \backslash\{v\}, \quad E\left(C^{\prime}\right)=E(C) \backslash\left\{v^{-} v, v v^{+}\right\} \cup\left\{v^{-} v^{+}\right\}
$$

We will use the set $V(C)$ as the set $H$ input into the ALGORITHM. Before we present the initial colouring of the graph $G[V(C)]$, we present some important observations about the considered vertex set which will be useful in the later part of the proof.

Claim 11. Vertex $v$ is the only neighbour of both $v^{-}$and $v^{+}$outside of $C^{\prime}$.

Proof. Otherwise, call the other such vertex $v^{\prime}$. Since $C$ is the longest cycle in $G$ and the graph $G$ is claw-free, then $v$ is adjacent to the other neighbour of $v^{-}$on $C$, call it $v^{--}$. The cycle $v v^{--} C v^{+} v^{\prime} v^{-} v$ (cp. Figure 1$)$ is a cycle longer than $C$, a contradiction.

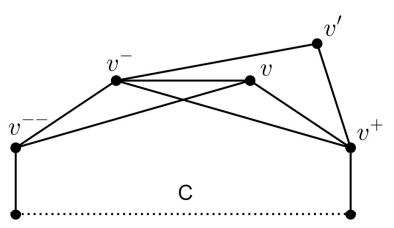

Figure 1: The longer cycle in Claim 11.

Claim 12. The vertex $v$ is not adjacent to all of the vertices of $C$.

Proof. Assume to the contrary that $v$ is adjacent to all vertices of the cycle $C$. By 2connectivity of $G$ there exists a path $P$ connecting the vertex $w$ to the cycle $C$ that does not include the vertex $v$. Denote by $w^{\prime}$ the end-vertex of $P$ which is a vertex of $C$. The neighbours of $w^{\prime}$ on $C$ we denote with respect to the considered orientation of $C$ by $w^{\prime-}$ and $w^{\prime+}$. Then the cycle $v w P w^{\prime} w^{\prime+} C^{\prime} w^{\prime-} v$ (cp. Figure 2) is clearly longer than the cycle $C$, which contradicts our assumption.

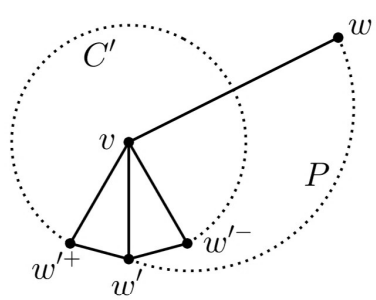

Figure 2: The longer cycle in Claim 12.

We consider all vertices of $C$ non-adjacent to $v$. We choose the vertex $u$ such that the distance on the cycle $C$ of $u$ to $v^{-}$or $v^{+}$is the smallest over all considered vertices. By Claim 12, such a vertex exists. Without loss of generality, we assume that $\operatorname{dist}_{C}\left(u, v^{-}\right)<$ 
$\operatorname{dist}_{C}\left(u, v^{+}\right)$. Notice that the vertex $u^{\prime} \in V(C) \operatorname{such}$ that $\operatorname{dist}_{C}\left(u^{\prime}, v^{-}\right)=\operatorname{dist}_{C}\left(u, v^{-}\right)$has to be connected by an edge to $v$, or else it would have been chosen as $u$.

Claim 13. There exists a vertex $s \in V(C)$ such that $N(s) \subseteq V(C)$.

Proof. Assume to the contrary that all vertices of the cycle $C$ have a neighbour outside of $C$. Thus, from maximality of $C$, every pair of vertices of the cycle at distance two on $C$ is connected by an edge. We denote by $a P^{2}(C) b$ a path connecting vertices $a, b \in V(C)$ that consists only of the edges between vertices of $C$ that are at distance two on the cycle $C$. Since $G$ is 2-connected, there exists a path $Q$ from $w$ to another vertex of the cycle $C$, say $v_{i}$. We will show that there exists a cycle longer than $C$ in the graph $G$. If the distance $\operatorname{dist}_{C}\left(v, v_{i}\right)$ is odd, then the cycle $v w Q v_{i} P^{2}(C) v^{+} C v_{i}^{-} P^{2}(C) v$ (cp. Figure 3 ) is longer than $C$. If $\operatorname{dist}_{C}\left(v, v_{i}\right)$ is even, we consider two cases. Both these cases are represented on Figure 4. If $w v^{--} \in E(G)$, then $v w v^{--} v^{-} v^{---} C v$ is again a longer cycle than $C$. If $w v^{--} \notin E(G)$, then $v^{--} v^{+} \in E(G)$ and the cycle $v w Q v_{i} P^{2}(C) v^{--} v^{+} C v_{i}^{-} P^{2}(C) v^{-} v$ is longer than $C$. In all of the cases we get a contradiction with maximality of $C$.

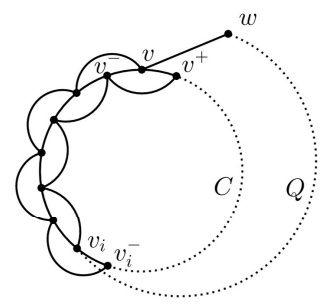

Figure 3: The longer cycle in Claim 13 when $\operatorname{dist}_{C}\left(v, v^{-}\right)$is odd.
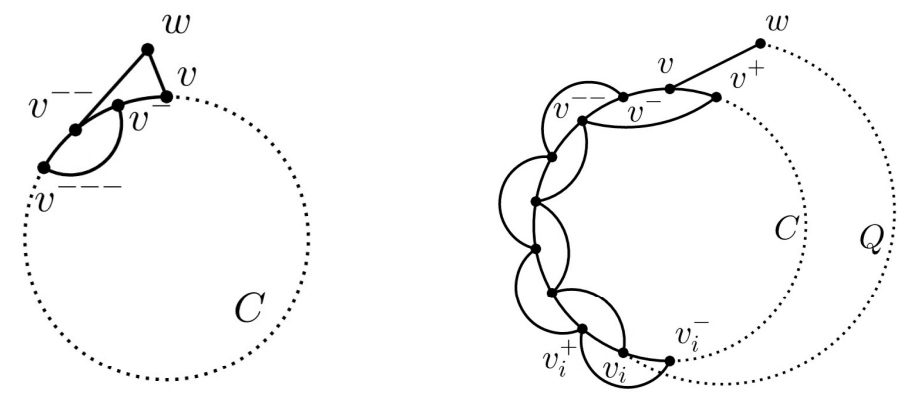

Figure 4: The longer cycles in Claim 12 when $\operatorname{dist}_{C}\left(v, v^{-}\right)$is even.

We are ready to present the colouring of the edges of the graph $G[V(C)]$. Colour the edges of the cycle $C^{\prime}$ and the edge $v^{-} v$ with colour 2 and the remaining edges of $G[V(C)]$ colour with 1. All assumptions for the input set $H=V(C)$ are fullfilled. In particular, by Claim 13, there exists a vertex $s \in V(C)$ such that $N(s) \subseteq V(C)$. We therefore use the ALGORITHM with input $H=V(C)$ to colour the remaining edges of the graph $G$. Let $c^{\prime}$ be the resulting colouring of $E(G)$. 
By Observation 7, the cycle $C^{\prime}$ is the only cycle that contains no edges coloured with colour 1. Therefore, the set $V(C)$ is fixed setwise. By Claim 11, the vertex $v$ is the only vertex of $G$ which has two neighbours that are consecutive vertices on the cycle $C^{\prime}$. Thus $v$ is fixed and also $v^{-}$is fixed as it is the only vertex of $C$ joined to $v$ by an edge coloured with colour 2. This breaks all of the rotations of $C$ and almost all of the symmetries. The only automorphism that remains for consideration is a symmetry of $C$ where $v^{-}$is a fixed point. To see that it is also broken, we use the fact that there exist vertices $u \in V(C)$ and $u^{\prime} \in V(C)$ such that $\operatorname{dist}_{C}\left(u^{\prime}, v^{-}\right)=\operatorname{dist}_{C}\left(u, v^{-}\right)$and $u v \notin E(G)$ while $u^{\prime} v \in E(G)$. Hence, for each $v \in V(C)$ we obtain that $v$ is fixed by the colouring $c^{\prime}$. By Theorem 8 , every vertex of the graph $G$ is fixed. Which ends the proof in this case.

We now consider the case where $G$ in not 2-connected. We consider the block and cut-vertex tree of the graph $G$. Let $B$ be a block that corresponds to a leaf in the block and cut-vertex tree and $v$ be the unique cut-vertex in this block. Denote by $u$ a neighbour of $v$ in $B$. Since $u$ is not a cut-vertex of the graph $G$, then the graph $G-u$ is connected and claw-free.

If the vertex $v$ has no neighbours in the block $B-u$ in the graph $G-u$, then the set $N(v)$ can be covered by a single path. We colour the edges of this path, as well as the edge from $v$ to the first vertex of this path, with colour 2 . If there is at least one neighbour of the vertex $v$ in $B-u$ in the graph $G-u$, then the neighbourhood of $v$ can be covered by two paths. We colour the edges of both of these paths, as well as the edges between the first vertices of these paths and $v$, with colour 2 . In both considered cases, we colour the remaining edges of $G[N(v)]$ with colour 1 . We obtain the colouring of the remaining edges of $G$ by inputting the graph $G-u$ with the set $H=N_{G-u}[v]$ into the ALGORITHM. To obtain the final colouring of the graph $G$, we colour all of the edges incident with $u$ with colour 1 .

The resulting colouring of the edges of the graph $G$ breaks every nontrivial automorphism of $G$. Firstly, by Observation 6 vertex $u$ is the only vertex only incident with edges coloured 1 , thus it is fixed. The vertex $v$ is the only cut-vertex adjacent to $u$, thus it is also fixed. Consider the first vertices of the paths in the minimal path cover of $N(v)$, call them $x_{1}$ and $x_{2}$ (possibly there is only one such vertex). They are the only not yet fixed vertices of $G$ joined to $v$ by an edge coloured with 2 . Let $B^{\prime}$ be the other block of the block and cut-vertex tree containing $v$. Any automorphism interchanging $B$ with $B^{\prime}$ is broken because $B^{\prime}$ does not contain a vertex incident with only edges coloured with 1 . Thus the vertices $x_{1}$ and $x_{2}$ are fixed. Moreover, all the other vertices of the paths in the minimal path cover of $N(v)$ are fixed. By a reasoning identical to the one presented in the proof of Theorem 8, we conclude that all of the remaining vertices of the graph $G$ are fixed.

\section{General Case}

In this section we prove Theorem 3 for $s \geqslant 4$. We begin by stating the following result due to Pilśniak, which will greatly shorten our proof. 
Theorem 14. [6] Let $G$ be a connected graph that is neither a symmetric nor a bisymmetric tree. If the maximum degree of $G$ is at least 3 , then

$$
D^{\prime}(G) \leqslant \Delta(G)-1
$$

unless $G$ is $K_{4}$ or $K_{3,3}$.

We now present our result together with the proof.

Theorem 15. Let $s \geqslant 4$ and let $G$ be a connected $K_{1, s}$-free graph with at least six vertices. Then

$$
D^{\prime}(G) \leqslant s-1
$$

Proof. If $\Delta(G)=2$, then $G$ is either a path or a cycle and $D^{\prime}(G)=2<s-1$. If $3 \leqslant \Delta(G) \leqslant s$, then the proof follows from Theorem 14. Assume then that $\Delta(G)>s$.

Let $x$ be a vertex such that $d_{G}(x)=\Delta(G)$. We use Theorem 8 and the ALGORITHM with $N[x]$ as the set $H$. Consider a minimal path cover $\mathcal{P}$ of $G[N(x)]$. Our task is to find a colouring $c$ of $G[N[x]]$, such that in the colouring $c^{\prime}$ obtained after application of the ALGORITHM every vertex of $N[x]$ is fixed.

As $\Delta(G)>s$, we need to consider only two cases.

Case 1. Assume that $\mathcal{P}$ contains a path with at least three vertices. Without loss of generality, let it be $P_{1}$. Colour the edges of this path, as well as the edge between the first vertex of this path and $x$ with 2 . Colour the edges between $x$ and the remaining vertices of this path with 3 . For each $i \neq 1$ colour the edges of $P_{i}$ as well as the edge between the first vertex of $P_{i}$ and $x$ with colour $i$. Colour with 1 every edge not yet coloured. This colouring uses at most $s-1$ colours (cp. Figure 5).

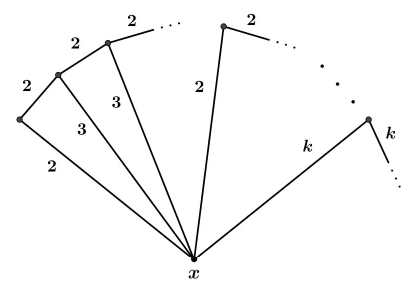

Figure 5: Case 1 of Theorem 15.

Now apply the ALGORITHM. If $P_{1}$ contains at least four vertices, then $x$ is the only vertex in $G$ contained in three triangles without edges coloured with 1 , therefore $x$ is fixed. If $P_{1}$ contains exactly three vertices, then $x$ and the second vertex of $P_{1}$, call it $x_{12}$, are the only vertices contained in two triangles without edges coloured with 1 . Vertex $x$ is adjacent to at least two edges coloured with 3 and at most two edges coloured with 2. If $x_{12}$ is adjacent to two edges coloured with 3 , then it is also adjacent to three edges coloured with 2 . Thus both $x$ and $x_{12}$ are fixed.

The first vertex of $P_{1}$ is fixed, because it is the only vertex joined to $x$ by an edge coloured with 2 and contained in the triangle without edges coloured with 1 . The second 
vertex of $P_{1}$ is fixed, because it is the only vertex joined to the first vertex of $P_{1}$ by an edge coloured with 2 and adjacent to $x$. Analogously, every vertex of the path $P_{1}$ is fixed.

Now, consider each path $P_{i}$ for $i \geqslant 2$. The first vertex of such a path is fixed, because it is the only not yet fixed vertex connected to $x$ by an egde coloured with $i$. Following the same argument as above, all the vertices of the path are fixed. Thus every vertex of $N[x]$ is fixed. By Theorem 8, every vertex of $G$ is fixed.

Case 2. Assume that $\mathcal{P}$ contains no path with three or more vertices. Therefore, $\mathcal{P}$ contains at least two paths with two vertices each. Without loss of generality, let $\left|P_{1}\right|=\left|P_{2}\right|=2$. We call their vertices $v_{1}, u_{1}$ and $v_{2}, u_{2}$, respectively. We colour the edges $v_{1} x, v_{2} x, v_{1} u_{1}$ with 2 and the edges $u_{1} x, u_{2} x, v_{2} u_{2}$ with 3 . For each $i \notin\{1,2\}$ consider the path $P_{i}$ and colour its edges and the edge joining the first vertex of this path and $x$ with $i-1$. Colour with 1 every edge not yet coloured. This colouring uses at most $s-1$ colours (cp. Figure 6).

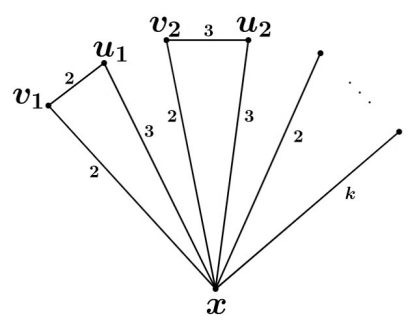

Figure 6: Case 2 of Theorem 15.

Now apply the ALGORITHM. Observe that $x$ is the only vertex in $G$ contained in two triangles without edges coloured with 1 . Thus vertex $x$ is fixed.

If $x$ is fixed, then for each nontrivial automorphism, the image of the vertex from $P_{1} \cup P_{2}$ is contained in $P_{1} \cup P_{2}$. The image of $v_{1}$ cannot be anything else than itself, as it is the only vertex in $P_{1} \cup P_{2}$ with two incident edges with colour 2 in $G[N[x]]$. Similarly, the vertex $u_{2}$ is fixed. Thus vertices $u_{1}$ and $v_{2}$ are fixed.

Now consider a path $P_{i}$ for $i \notin\{1,2\}$. It consists of at most two vertices. These vertices are fixed, because the first one is the only not yet fixed vertex joined to $x$ by an egde coloured with $i-1$, and the second one (if it exists) is the only vertex adjacent to $x$ and adjacent to the first vertex by an egde coloured with $i-1$. Thus every vertex of $N[x]$ is fixed. By Theorem 8, every vertex of $G$ is fixed.

\section{Acknowledgments}

We want to sincerely thank Monika Pilśniak for all the effort she put into helping us write this paper.

\section{References}

[1] M. O. Albertson and K. L. Collins, Symmetry breaking in graphs, Electron. J. Combin. 3 (1996) \#R18. 
[2] R. Diestel, Graph Theory, 5th Edition, Springer-Verlag, Heidelberg, 2016.

[3] R. Faudree, E. Flandrin and Z. Ryjáček, Claw-free graphs - a survey, Discrete Math. 164 (1997), 87-147.

[4] J.L. Fouquet, A strengthening of Ben Rebea's lemma, J. Combin. Theory Ser. B 59 (1993) 35-40.

[5] R. Kalinowski and M. Pilśniak, Distinguishing graphs by edge colourings, European J. Combin. 45 (2015), 124-131.

[6] M. Pilśniak, Improving Upper Bounds for the Distinguishing Index, Ars Math. Contemp. 13 (2017) 259-274. 